\title{
Growth-based 3D modelling using stem-voxels encoded in digital-DNA structures
}

\author{
THOMAS RAYMOND, Kingston University, London \\ VLADISLAV LI, Kingston University, London \\ VASILEIOS ARGYRIOU, Kingston University, London
}

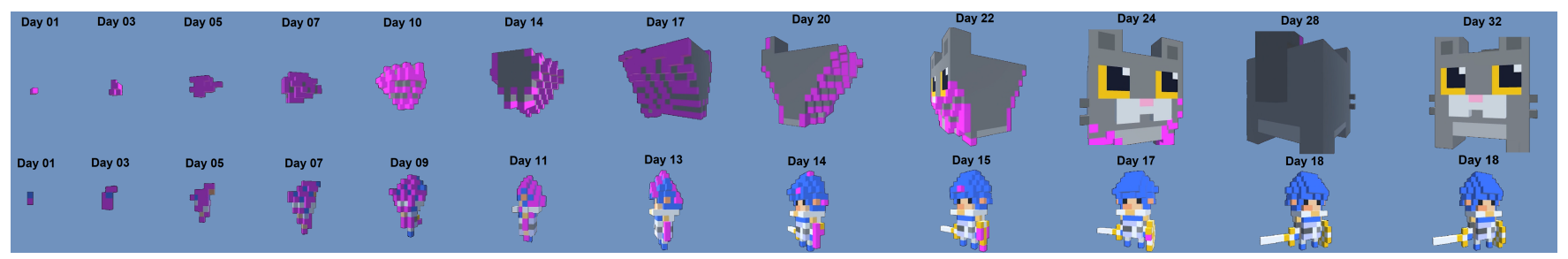

Fig. 1. Growth based 3D modelling from a single stem-voxel to a full 3D structure.

Leaps in technology are increasingly making the prospect of using biological structures as part of digital models and artwork a tangible reality. In this work a new method heavily inspired by natural biological processes for 3D modelling and animation is proposed. The proposed approach differentiates from the classic assembly or printing methodologies and offers a novel growth based solution for the design and modelling of 3D structures. In order to facilitate the needs of growth based modelling, new terms and graphic primitives such as stem-voxels, muscle-voxels, bone-voxels and digital-DNA are introduced. The core production rules of a novel context free grammar were implemented allowing 3D model designers to build the digital-DNA of a 3D model that the introduced parser will interpret to a full 3D structure. The obtained 3D models support animation using the musclevoxels, they are able to observe the environment using photoreceptor-voxels and interact with a level of intelligence based on neural networks build with nerve-voxels. The proposed modelling solution was evaluated with a variety of volumetric models demonstrating a strong potential and impact, with many applications and offering a new tool for 3D modelling systems.

Additional Key Words and Phrases: 3D modelling, growth based modelling, stem-voxels, digital-DNA

ACM Reference Format:

Thomas Raymond, Vladislav Li, and Vasileios Argyriou. 2020. Growth-based 3D modelling using stem-voxels encoded in digital-DNA structures. In SIGGRAPH Asia 2020 (SA '20 Posters), December 04-13, 2020. ACM, New York, NY, USA, 2 pages. https://doi.org/10.1145/3415264.3425443

\section{INTRODUCTION}

Research into taking inspirations from biology and integrating them with digital 3D models has been ongoing over the last decades offering solutions on human body modelling, motion analysis and breathing or muscle simulation [Jehee and Hoon [n.d.]; Tsoli et al. 2014]. Bioinspired modelling and design has wide range of practical

Permission to make digital or hard copies of part or all of this work for personal or classroom use is granted without fee provided that copies are not made or distributed for profit or commercial advantage and that copies bear this notice and the full citation on the first page. Copyrights for third-party components of this work must be honored. For all other uses, contact the owner/author(s).

SA '20 Posters, December 04-13, 2020, Virtual Event, Republic of Korea

(C) 2020 Copyright held by the owner/author(s).

ACM ISBN 978-1-4503-8113-0/20/11.

https://doi.org/10.1145/3415264.3425443 applications and it is considered an important problem. One way that biology creates an impact on modelling and animation is that offers the basis for realism in motion synthesis considering the actual dynamics of muscle activations and response times [Jiang et al. 2019]. Creating realistic behaviours of a human musculoskeletal system of virtual avatars plays an integral role in bringing fictional characters to life in films and games, [Clegg et al. 2018].

In computer graphics the design and modelling processes follows in most of the cases the assembly line approach. Parts and components are modelled separately with the final item to be a composition of them. Considering a 3D car model, it is composed of the main body, the doors, the wheels, the engine, and glass windows, that all can be designed independently having different materials or properties. At later stage rigging and animation are applied to allow the model to perform movements and actions. Finally, when the model is integrated in game or an animated movie, a logic is applied in the form of a state machine providing a level of intelligence.

In this work, we propose a novel bioinspired pipeline for 3D modelling, animation and intelligence that does not follow the assembly line approach but is based on the same principles that are followed by the living organisms. A new digital-DNA representation and growth-based approach is introduced offering both modelling and animation bioinspired solutions providing high compactness and efficiency in comparison with current standards and solutions.

\section{METHODOLOGY FOR GROWTH-BASED MODELLING}

The proposed modelling and animation approach is inspired from the mechanisms used in living multicellular organisms to represent, store and interpret the required information that lead to their creation (or birth) starting from a single cell. All the required information that is essential to create a living organism replicating all the steps from the moment of conception to birth is stored in DNA. Based on these principles and considering the set of cell functionalities, the proposed modelling method is following the same principles of division and differentiation as all multicellular organisms.

At this stage we need to introduce few new concepts that will allow us to apply the functions of living cells to the proposed 3D 
growth-based modelling solution. We consider a voxel to represent a primitive element (cell) but other primitives could be considered too. For the proposed primitive element we consider the following five modalities a) stem-voxel, b) bone-voxel, c) muscle-voxel, d) nerve-voxel, and e) photoreceptor-voxel. The above voxel modalities have common properties and specializations. In more details all the voxel types can be divided producing two children voxels, and the modality of the children is the same with the parent voxel for all the cases, except the stem-voxels, that can generate children of any modality. Furthermore, all the voxel modalities have a texture (or colour) property.

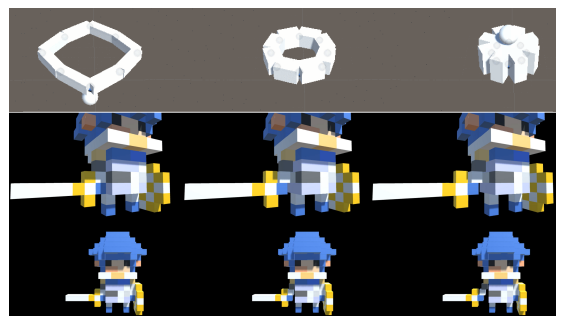

Fig. 2. Example of muscle-voxels contracting and relaxing.

About the specializations, stem-voxels have the ability to differentiate (i.e. produce children of any type). This means that a stem-voxel can produce either other stem-voxels or differentiated ones, or combination of them. The bone-voxels do not have any specialisations and their main purpose is to construct and hold the body of a 3D model. Muscle-voxels are able to contract and relax in one direction, perpendicular to one of their six faces and be connected to another muscle or bone-voxel (see figure 2). A nerve-voxel represents a neuron, storing a weight value and can be connected with multiple nerve-voxels, or linked to muscle- and photoreceptorvoxels, activating them or receiving input, respectively. Finally, each photoreceptor-voxel can cast a ray to a direction perpendicular to one of its faces allowing to perceive the RGB colour of any intersected object. The photoreceptor-voxels can be connected to nerve-voxels providing input to them. The proposed novel modelling method is not following the assembly line approach or the $3 \mathrm{D}$ printing one that is placing different voxels at certain locations. Instead, a growth based modelling approach is introduced starting from a single stem-voxel that after a number of divisions and differentiations produces a final 3D model. All the information regarding the divisions and differentiations is encoded in a structure that represents the digital-DNA (d-DNA) of a given model. The introduced d-DNA structure includes all the information for the divisions and differentiations plus discrete time information indicating when each one of the actions will occur.

One of the main contributions of this work is the core interpreter (parser) that was designed and implemented, allowing the interpretation of the proposed d-DNA structure to a growing 3D model based on the principles of division and differentiation. A context free grammar was designed to facilitate the parser requirements with the list of the terminal and nonterminal symbols to be shown in table 1 . The proposed grammar for 3D model growth includes a set of production rules, that describe all possible divisions and differentiations using simple replacements. A set of additional productions rules have been designed and implemented to accommodate voxel shifting, when collisions occur after the division process. The proposed novel grammar includes the time element that allows the voxels to know when to divide or differentiate. Finally, special terminal elements are used to represent the additional properties of the specialised voxels, such as the muscle connection, the ray-cast direction of the photoreceptor and the weights of the nerve-voxels. More details are available at the video in the supplementary materials.

Table 1. Sample of Terminal/ Nonterminal symboles and Production rules

\begin{tabular}{l|l} 
Terminal & Nonterminal \\
\hline bv, mv, nv, & O: Organism, V: Set of Voxels, SVi: Stem \\
pv, +x,-x,+y,- & Voxel, BVi: Bone Voxel, MVi: Muscle Voxel, \\
y,+z,-z & NVi: Nerve Voxel, PVi: Photoreceptor Voxel \\
\hline Production rules \\
\hline S0->O; O->Vi| OVi; Vi->SVi| BVi| MVi| NVi| PVi; SVi- \\
>SVi SVi| SVi BVi| SVi MVi|SVi NVi|SVi PVi|BVi SVi|MVi \\
SVi|NVi SVi|PVi SVi| BVi BVi| MVi MVi| NVi NVi| PVi PVi| \\
bv|mv|nv|pv; BVi-> BVi BVi|bv; MVi-> MVi MVi|mv; NVi-> \\
NVi NVi|nv; PVi->PVi PVi| pv; i-> +x,-x,+y,-y,+z,-z \\
\hline
\end{tabular}

\section{RESULTS AND CONCLUSIONS}

The proposed growth based modelling approach was tested with several volumetric models and examples are shown in figure 1. A variety of scenarios are supported allowing the combination of multiple voxel types in a single model. The developed parser and digital-DNA designing and editing tool will become available online.

This work is still at an early stage, but the potential and impact of growth-based modelling is high with significant applications allowing the designers to incorporate in a single structure shape, animation, environment observation and intelligence. Furthermore by editing only a small percentage of the digital-DNA, it will allow to generate similar variations of a given model. Additionally medical imaging applications that process volumetric data such as 3D MRI will be able to better understand the shape and behaviour of human organs. Finally, games and virtual worlds like Minecraft could benefit from growth-based 3D modelling by supporting autonomous agents and novel growth-based structures using digital-DNA.

\section{REFERENCES}

Alexander Clegg, Wenhao Yu, Jie Tan, C. Karen Liu, and Greg Turk. 2018. Learning to Dress: Synthesizing Human Dressing Motion via Deep Reinforcement Learning. ACM Trans. Graph. 37, 6 (2018), Article 179.

J.Lee Jehee and LeeKang Hoon. [n.d.]. Precomputing Avatar Behavior from Human Motion Data. ([n.d.]).

Y. Jiang, T. Wouwe, F. DeGroote, and C. Liu. 2019. Synthesis of biologically realistic human motion using joint torque actuation. ACM Tran Graphics 38 (2019), 1-12.

A. Tsoli, N. Mahmood, and M.J. Black. 2014. Breathing Life into Shape: Capturing, Modeling and Animating 3D Human Breathing. ACM SIGGRAPH (2014). 